\section{RELATIONSHIP OF APNEIC PAUSE DURATION AND AIRWAY PROTECTION IN PEDIATRIC PATIENTS REFERRED FOR VIDEOFLUOROSCOPIC SWALLOWING STUDIES}

\author{
C.K. Miller ${ }^{1,2}$, L.N. Kelchner ${ }^{2,3}$
}

${ }^{1}$ Cincinnati Children's Hospital Aerodigestive Center, ${ }^{2}$ Division of Speech Pathology, Cincinnati Children's Hospital, ${ }^{3}$ Communication Sciences and Disorders, University of Cincinnati, Cincinnati, $\mathrm{OH}, \mathrm{USA}$

Background and aims: Coordination of breathing and swallowing must be precise for maintenance of airway protection and prevention of aspiration. The phases of respiration are interrupted during swallowing; swallowing apnea must be of sufficient duration to protect the airway to prevent entry of food or liquid. The specific respiratory phase patterns and the association with normal and abnormal swallowing parameters in pediatric patients are presently unknown. The aim of the present project was to analyze the duration of apneic pause during swallowing in association with swallowing parameters in a cohort of pediatric patients undergoing videofluoroscopic studies of swallowing.

Methods: Acohort of 50 pediatric patients undergoing videofluoroscopic swallowing studies was identified. The KayPentax Swallowing Signals Lab was used to digitally record the swallow studies concurrently with the respiratory signals. Nasal respiratory airflow was captured using a standard nasal cannula coupled to the video recording device.

Results: Variation was noted across subjects in regard to respiratory phase pattern associated with bolus type (thin liquid, thickened liquid, or solid). Variability in swallowing apnea duration was noted across all subjects, however, a significant difference in relation to the duration of swallowing apnea was found in participants who demonstrated aspiration.

Conclusions: Participants who did not display swallowing dysfunction were noted to demonstrate increased swallowing apnea duration in comparison to the participants who had aspiration events. Continued research is needed to delineate the relationship between respiratory phase patterns and swallowing abnormalities. Such data will assist clinicians in diagnosis and implementation of appropriate compensatory treatment strategies.

\section{O AUDIT OF THE USE OF CEREBRAL FUNCTION MONITORING (CFM) IN THE MANAGEMENT OF TERM NEONATES WITH ENCEPHALOPATHY}

\section{J.O. Menakaya}

\section{Department of Neonatal Paediatrics, Hillingdon Hospital NHS Trust, Uxbridge, UK}

Objectives: To determine the indications for cerebral function monitoring, the characteristics of babies who had CFM and their outcomes at discharge.

Methods: The records of all term newborn babies born between June 2006 - April 2009 who underwent cerebral function monitoring were reviewed.

Results: Sixty six term $(55 \%$ male $)$ babies underwent cerebral function monitoring. Their mean GA was $39.2 \pm 1.6 \mathrm{wk}$. The average birth weight was $3.09 \pm 0.75 \mathrm{~kg} .58 \%$ of these babies were delivered operatively. The apgar scores were 4t 3@1min, $6.2 \pm 2.8 @ 5 \mathrm{~min}$ and $7 \pm 2.6 @ 10 \mathrm{~min} .46 \%$ of these babies were intubated at birth. 43\% of the babies had clinical seizures. 3 groups of babies were identified: Group 1- 11 babies had both the lower margin of CFM trace less than $5 \mu \mathrm{V}$ and the upper margin less than $10 \mu \mathrm{V}$. Group 2- 8 babies had the lower margin less than $5 \mu \mathrm{V}$ but upper margin higher than $10 \mu \mathrm{V}$. Group 3 - 46 babies had appropriate voltages on CFM tracing. 14 babies from groups $1 \& 2$ had moderate or severe encephalopathy and 2 had normal neurological signs at discharge. Normal CFM tracing in encephalopathic babies obviated the need for neuro-protective treatment. CFM was useful in evaluating encephalopathic babies from other causes. These included persistent hypoglycaemia, cortical haemorrhage, periventricular echodensities, kernicterus, propionic acidaemia, middle cerebral artery infarct, generalised hypotonia of unknown cause and moebius syndrome.

Conclusion: Majority of babies with encephalopathy had a CFM trace with normal voltages. It is an important tool in the clinical assessment of term neonates with encephalopathy. 